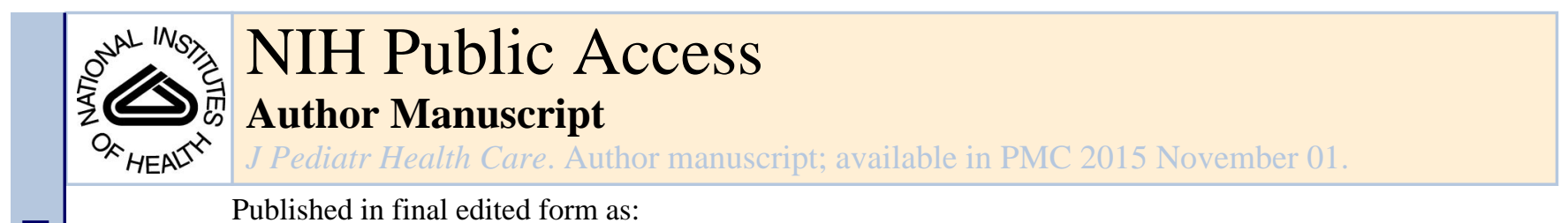

Published in final edited form as:

J Pediatr Health Care. 2014 ; 28(6): 541-549. doi:10.1016/j.pedhc.2014.05.003.

\title{
HPV vaccine hesitancy: Findings from a statewide survey of healthcare providers
}

\author{
Annie-Laurie McRee, $\mathrm{DrPH}^{1}$, Melissa B. Gilkey, $\mathrm{PhD}^{2}$, and Amanda F. Dempsey, MD, PhD, \\ $\mathrm{MPH}^{3}$ \\ ${ }^{1}$ Division of Health Behavior and Health Promotion, College of Public Health, The Ohio State \\ University (Columbus, $\mathrm{OH}$ ) \\ ${ }^{2}$ University of North Carolina Lineberger Comprehensive Cancer Center (Chapel Hill, NC) \\ ${ }^{3}$ Children's Outcomes Research Program, University of Colorado Denver (Denver, CO)
}

\section{Abstract}

Introduction-Healthcare provider recommendations are critical for human papillomavirus (HPV) vaccine uptake. We sought to describe providers' HPV vaccine recommendation practices and explore their perceptions of parental hesitancy.

Method-A statewide sample $(n=575)$ of Minnesota healthcare providers ( $20 \%$ pediatricians, $47 \%$ family medicine physicians, $33 \%$ nurse practitioners) completed our online survey in April 2013.

Results-Only $76 \%$ of healthcare providers reported routinely recommending HPV vaccine for girls ages 11-12, and far fewer (46\%) did so for boys ( $p<.001)$. A majority of providers reported asking questions about parents' concerns (74\%), but many lacked time to probe reasons (47\%) or felt that they could not change parents' minds (55\%). Higher levels of self-efficacy and outcome expectations were associated with routine recommendations ( $p<.05)$.

Discussion-Findings suggest that providers' perceptions of hesitancy may discourage them from routinely recommending HPV vaccine. Improving providers' self-efficacy to address hesitancy may be important for improving vaccination rates.

\section{Keywords}

HPV vaccine; vaccine hesitancy; healthcare provider communication

(C) 2014 National Association of Pediatric Nurse Associates and Practitioners. Published by Mosby, Inc. All rights reserved. Correspondence to: Annie-Laurie McRee, Division of Health Behavior and Health Promotion, College of Public Health, The Ohio State University, 354 Cunz Hall, 1841 Neil Ave, Columbus, OH 43210, almcree@ @ph.osu.edu, Phone: (614)292-4216.

Potential conflicts of interest: ALM and MBG have no conflicts of interest to disclose. AFD serves on an advisory board for Merck, a company that manufactures HPV vaccine, though she does not receive research funding from this company.

Publisher's Disclaimer: This is a PDF file of an unedited manuscript that has been accepted for publication. As a service to our customers we are providing this early version of the manuscript. The manuscript will undergo copyediting, typesetting, and review of the resulting proof before it is published in its final citable form. Please note that during the production process errors may be discovered which could affect the content, and all legal disclaimers that apply to the journal pertain. 
Human papillomavirus (HPV) vaccine is a cost-effective way to confer protection against genital warts and several types of cancer, and yet it remains one of the most underused vaccines in the United States. National guidelines have recommended routine administration of HPV vaccine to 11-12 year old females since 2006 (Markowitz et al., 2007). In October 2011, this recommendation was extended to males (Dunne et al., 2011), strengthening the previous "permissive recommendation" that HPV vaccine received in 2009 (Centers for Disease Control and Prevention (CDC), 2010). Despite these recommendations, estimates from 2012 indicate that only $33 \%$ of adolescent girls and $7 \%$ of boys have completed the 3dose HPV vaccine series (Curtis et al., 2013). By contrast, two other vaccines introduced to adolescents' immunization schedule in the same time period have made steady progress toward the Healthy People 2020 goal of $80 \%$ coverage (U.S. Department of Health and Human Services). In light of disappointing uptake, research to understand the unique challenges of HPV vaccination has gained prominence.

Of the many factors that influence HPV vaccination, the role of healthcare providers is perhaps the most important. Receiving a providers' recommendation is one of the strongest and most consistent predictors of HPV vaccination (Allen et al., 2010; Reiter, Brewer, Gottlieb, McRee, \& Smith, 2009; Rosenthal et al., 2011; Ylitalo, Lee, \& Mehta, 2013). Despite evidence suggesting that they are highly influential, healthcare providers often fail to recommend the vaccine according to guidelines (Vadaparampil et al., 2011) Parental reports suggest that only about half of female and 1 in 10 male adolescents have ever received a recommendation for HPV vaccine (Darden et al., 2013; Reiter, Gilkey, \& Brewer, 2013) and lack of provider recommendation is one of the most common reasons parents give for not getting the vaccine for their child (Dorell, Yankey, \& Strasser, 2011; Reiter, Katz, \& Paskett, 2013).

Healthcare providers face a number of barriers to recommending HPV vaccine, some of which are interpersonal in nature. For example, some providers express difficulty discussing sexual health issues that may attend HPV vaccination, particularly with younger adolescents in the target age range for vaccination (Daley et al., 2010; Vadaparampil et al., 2011). Others perceive parents as being opposed to the vaccine and are reluctant to strongly recommend the vaccine due to concerns about initiating time-consuming or confrontational debates (Daley et al., 2010; Hughes, Jones, Feemster, \& Fiks, 2011). Indeed, prior research suggests that vaccine refusal and delay is common among parents of adolescents (Daley et al., 2010; Gilkey, McRee, \& Brewer, 2013), although relatively little is known about how providers understand or address parental hesitancy specific to HPV vaccine. We sought to describe healthcare providers' HPV vaccine recommendation practices and to explore their perceptions of, and approaches to addressing, HPV vaccine hesitancy among parents of 11to 12-year-old youth.

\section{Methods}

We surveyed a statewide sample of physicians and nurse practitioners who provide preventive care to preteens and adolescents ages 11-17 years. We identified potential participants through publically available lists provided by the Minnesota Boards of Medical Practice and of Nursing. From these lists, we sampled healthcare providers in pediatric and 
family medicine specialties. Because our sampling frame included many providers who potentially did not meet study criteria (e.g., neonatal specialists), the survey used a screener question to limit respondents to those who provided preventive care to patients ages 11-17 years.

In April 2013, we invited 3,923 healthcare providers to participate in the study. A total of 615 respondents provided informed consent, and took our cross-sectional, online survey (adjusted response rate based on AAPOR formula 4: 28\%) (American Association for Public Opinion Research, 2009). Among those who were confirmed to be eligible based on survey screening questions, the cooperation rate was $86 \%$ (i.e., the proportion of eligible providers contacted who completed the survey; AAPOR, 2009). Study data were collected and managed using REDCap (Research Electronic Data Capture) tools hosted at the University of Minnesota (Harris et al., 2009). Respondents received a survey invitation describing the study's purpose and providing a link to the survey; non-respondents received up to 3 reminder emails. No incentives were provided for participation. The Institutional Review Board at the University of Minnesota approved the study.

\section{Measures}

We developed items based on the literature and our previous research with parents (Dempsey, Abraham, Dalton, \& Ruffin, 2009; Gilkey et al., 2013; Hughes et al., 2011; Kempe et al., 2011; Opel et al., 2012; Vadaparampil et al., 2011). We conducted cognitive interviews with 5 healthcare providers to identify potential sources of response error and ensure the clarity of new survey items (Willis, 2005). We used findings from cognitive interviews to refine and improve survey items before fielding the full survey. Questions specified the 11-12 year old age group to align with HPV vaccination guidelines from the Advisory Committee on Immunization Practices (ACIP) (Centers for Disease Control and Prevention (CDC), 2010; Dunne et al., 2011; Markowitz et al., 2007).

The survey assessed providers' HPV vaccine recommendation practices with 3 parallel items asked separately about girls and boys. The first question asked how often providers recommended HPV vaccine as a part of routine care for 11-12 year olds. We categorized responses for this analysis as "most of the time" (defined as "more than $75 \%$ of the time") vs. less often to reflect ACIP guidelines for routine administration (Centers for Disease Control and Prevention (CDC), 2010; Dunne et al., 2011; Markowitz et al., 2007) and to address the skewed nature of the distributions. Based on our formative qualitative research, the survey also included two statements assessing the strength of providers' HPV vaccine recommendations.

Following text instructing respondents to think about the times during the last year they recommended HPV vaccine to parents of 11-12 year olds patients, providers indicated how often they encountered different types of parental reactions (5 items) and rated how much potential concerns contributed to parents' decisions to delay or refuse HPV vaccine for their child (12 items). The survey then assessed providers' responses to parental HPV vaccine hesitancy (6 items), self-efficacy (i.e., confidence in their ability to address parents' concerns; 4 items), outcome expectations (i.e., expectations that their discussions with 
parents will lead to vaccination; 3 items) (Bandura, 1986), and perceptions of potential strategies and tools for counseling HPV-vaccine hesitant parents (8 items).

Respondents also provided information about sociodemographic and practice characteristics including: age, sex, training specialty, year finished clinical training, practice type and location, and proportion of patients 11-17 years old.

\section{Analyses}

Of the 615 total respondents, the analytic sample for the present analysis includes 575 respondents who answered questions about HPV vaccine recommendations and parental hesitancy. We used chi-square analyses to test for differences in recommendation practices by provider type (i.e., pediatrician, family medicine physician, or nurse practitioner). We assessed associations between routinely recommending HPV vaccine and providers: perceptions of parents' reactions, responses to hesitancy, self-efficacy to address parental concerns, and outcomes expectations using logistic regression models controlling for provider type. We conducted all analyses in Stata SE version 10.0 (Statacorp, College Station, TX) using two-tailed tests and a critical alpha of .05.

\section{Results}

\section{Respondent characteristics}

The average age of respondents was 48 years (Table 1). Most respondents were female (72\%). About half were family medicine physicians (47\%) and one-third were nurse practitioners $(33 \%)$. Most respondents worked in either private practice $(32 \%)$ or a practice network (31\%) and served patient populations comprised of more than $10 \%$ adolescents (60\%). Respondents included in the analysis did not differ from those excluded with regards to any of the assessed socio-demographic and practice characteristics (all $p>.05$ ).

\section{HPV vaccine recommendation practices}

About three-quarters of healthcare providers (76\%) reported recommending HPV vaccination to girls ages 11-12 as a part of their routine care "most of the time." Providers routinely recommended the vaccine for boys less often than for girls, with fewer than half $(46 \%)$ reporting that they do so "most of the time" $\left(\chi^{2}=152.49, p<.001\right)$. As shown in Figure 1 , more pediatricians routinely recommended HPV vaccine for boys than did both family medicine physicians and nurse practitioners (67\% vs. $42 \%$ and $41 \%$ respectively, $p<.001$ ). HPV vaccine recommendations for girls did not differ by provider type $(p=.06)$.

Many providers "somewhat" or "strongly" agreed that they recommend HPV vaccine as strongly as they do other adolescent vaccines (for girls, 75\%; for boys, 64\%). However, most also agreed that they prefer to offer HPV vaccine as an optional vaccine for girls (62\%) or for boys (69\%) ages 11-12.

\section{Parental reactions to HPV vaccine recommendations for 11-12 year olds}

One-third of providers (33\%) reported that parents frequently (i.e., "often" or "most of the time") accept HPV vaccine recommendations without question, while half (51\%) reported 
that parents frequently ask to delay vaccination (Table 2). One-fifth (18\%) indicated that parents frequently respond with concern about HPV vaccine, and $12 \%$ reported that parents frequently refuse to get HPV vaccine for their child. Providers had lower odds of recommending HPV vaccine to 11-12 years olds "most of the time" if they reported that parents responded more frequently with requests to delay vaccination or if they more frequently refused HPV vaccine for their child. Providers who reported that parents more frequently accepted HPV vaccine without question had higher odds of recommending it "most of the time" (all $p<.05$ ). Parental reactions of anger or concern about HPV vaccine were not statistically associated with providers' HPV vaccine recommendation practices.

In terms of reasons for HPV vaccine delay or refusal, most providers perceived factors that contributed "some" or "a lot" to parents' decisions to include the belief that their child is not sexually active (79\%) or is unlikely to get an HPV-related disease (63\%), as well as parental discomfort talking with their child about sex (55\%; Table 3). Providers perceived that concerns about short-term side effects, cost, or effectiveness contributed little to parents' decisions to delay or refuse HPV vaccination for their child.

\section{Provider responses to parental HPV vaccine hesitancy}

Most providers reported frequently (i.e., "often" or "most of the time") responding to parental HPV vaccine hesitancy by offering reassurance that the vaccine is safe (90\%), giving standard written information (such as the Centers for Disease Control and Prevention's Vaccine Information Sheets; 83\%), or asking questions to explore parents' concerns (74\%; Table 2). All assessed responses to parental HPV vaccine hesitancy were positively associated with recommending HPV vaccine to 11-12 year old girls or boys "most of the time" (all $p<.001$ ) with the exception of suggesting that parents delay vaccination until a future visit which was associated with lower odds of routine recommendation.

Most healthcare providers felt confident that they could address parental concerns about sexual disinhibition (86\% "somewhat agreed" or "strongly agreed"; Table 4) and HPV vaccine for boys (88\%). However, nearly half indicated that they did not have enough time during visits to probe parents' reasons for vaccine hesitancy (47\%). While the vast majority of providers felt that they are influential in parents' decisions about whether to get HPV vaccine for their child (91\%), two-thirds (55\%) felt that there was not much they could say to change the minds of parents who parents wish to delay or refuse vaccination. All assessed self-efficacy and outcome expectations beliefs were positively associated with routinely recommending HPV vaccine to 11-12 year olds (all $p<.05$ ) with the exception of believing that there is not much they can say to change parents' minds which was not statistically significant for either girls or boys.

\section{Tools and strategies for counseling HPV vaccine-hesitant parents}

The strategies and tools that providers perceived would be most helpful (i.e., "a lot" or "somewhat") for counseling parents who are hesitant to get their adolescent children vaccinated against HPV were: information tailored to specific parent concerns (74\%) or to parents' cultural background (68\%), and providing information about HPV vaccine to parents prior to the clinical visit (72\%; Table 3). Over half of providers also felt that it 
would be helpful to have a screening tool to identify specific parental concerns $(58 \%)$ or a discussion guide (57\%).

\section{Discussion}

Despite national guidelines for routine HPV vaccination of 11-12 year-olds, nearly 1 out of 4 healthcare providers in our statewide sample indicated that they are not routinely recommending the vaccine for girls, and more than half reported not doing so for boys even 18-months after the release of guidelines recommending routine vaccination of males. While such gender-based differences are not surprising given the initial roll-out of the vaccine for girls only, they are concerning. If trends in vaccine uptake among females provide an indication of trajectories for males, vaccine coverage may increase, but remain below targets. Furthermore, our findings suggest that recommendations providers do deliver may be ambiguous; most respondents reported preferring to offer the vaccine as optional, and a significant minority indicated that they do not recommend HPV vaccine as strongly as other adolescent vaccines. As receiving a provider's recommendation is one of the strongest correlates of HPV vaccination, our findings lend support to a growing body of literature that highlights the importance of increasing the frequency and quality of recommendations (Allen et al., 2010; Reiter et al., 2009; Rosenthal et al., 2011; Vadaparampil et al., 2011). Improving HPV vaccine recommendations may be especially important for raising coverage among boys since parents are less likely to know that the vaccine is available for their sons (Gilkey, Moss, McRee, \& Brewer, 2012).

Healthcare providers in our sample perceived parental HPV vaccine hesitancy to be common; about half of respondents reported that parents frequently request to delay or sometimes wish to refuse HPV vaccination. The high frequency of perceived hesitancy is concerning and, in aggregate, may constitute a substantial burden for providers, who experience vaccine hesitancy as a frustrating and time-consuming problem (Kempe et al., 2011). Importantly, our findings suggest that perceptions of parental hesitancy may discourage providers from delivering recommendations according to guidelines. This is particularly concerning as recent research suggests that providers are over-estimating parental concerns with regard to HPV and flu vaccines (Healy, Montesinos, \& Middleman, 2014). Providers may be reassured to learn that parental concerns about adolescent vaccines are not as frequent or severe as they suspect. Indeed, consistent with previous findings on HPV vaccine recommendations for females (Daley et al., 2010), providers in this sample who reported more frequently encountering parental responses of refusal or delay were less likely to routinely recommend HPV vaccination to either female or male adolescents.

Our findings suggest that providers' self-efficacy to address parental HPV vaccine hesitancy and their outcome expectations may also influence their recommendation practices. We found that respondents with lower levels of confidence in addressing concerns and who felt they were not influential or able to convince parents to get the vaccine were less likely to routinely recommended HPV vaccination to either males or females. Although the crosssectional nature of our data preclude drawing conclusions about the directionality of this relationship, prior research supports the hypothesis that providers' self-efficacy and outcome expectations are associated with adherence to clinical guidelines (Cabana et al., 1999). 
Improving providers' self-efficacy to address parental concerns may be important for supporting recommendation practices and ultimately improving HPV vaccine uptake in the target age group.

Respondents in our sample perceived parents' association between HPV vaccine and sexual activity to be an especially common source of hesitancy. Provider training or other tools may be particularly helpful if aimed at helping to convey that HPV vaccination is most beneficial before the onset of sexual activity and that HPV is highly prevalent. In addition, identifying ways of discussing HPV vaccine in a manner that helps providers navigate discomfort with discussing sexual activity may also be important, as these topics may not be adequately addressed in vaccine conversations (Perkins \& Clark, 2013) and providers may be less likely to recommend HPV vaccine if they feel they must broach sexuality in the context of HPV vaccine discussions (Daley et al., 2010). Our finding that very few healthcare providers perceived that parents react to HPV vaccine recommendations by being offended or angry may give providers more confidence in initiating these discussions.

To effectively communicate with vaccine-hesitant parents, healthcare providers need to understand parents' specific concerns. However, a quarter of providers in our sample reported that they only sometimes or rarely ask questions to explore parents' reasons, and many indicated lacking time to address parental hesitancy. Effective and efficient ways of assessing and addressing parents' vaccine concerns are needed, and many providers in this study indicated that a screening questionnaire to identify parental concerns would be helpful for counseling HPV-vaccine hesitant parents. Research suggests that such tools can be a reliable and valid way to identify parents who are hesitant to get their young children vaccinated (Opel et al., 2011), though to date, no such screener exists for vaccines in adolescent platform. Most providers also indicated that written information that addresses specific concerns would be helpful. Such targeted information may increase the relevance of information provided to parents (Kreuter \& Wray, 2003) and could be an effective and lowburden supplement to the standard written information that the vast majority already offers parents. Beyond didactic information which alone is unlikely to change parents' parents' intentions to vaccinate (Dempsey, Zimet, Davis, \& Koutsky, 2006; Lechuga, Swain, \& Weinhardt, 2012), providers in our sample were also interested in an interactive decision aid (Fiks et al., 2013) which could assist parents in making decisions about HPV vaccine before or during a clinical visit (e.g., in the waiting room). Future intervention research should explore these opportunities; available tools for early childhood immunization may offer a starting point for this work.

Study findings highlight differences in HPV vaccine recommendation practices based on provider specialty. In contrast to previous research (Daley et al., 2010; Weiss, Zimet, Rosenthal, Brenneman, \& Klein, 2010), we did not find provider type differences in HPV vaccine recommendations for girls. However, we did note differences in recommendations for boys with fewer family medicine physicians and nurse practitioners routinely recommending the vaccine $\mathrm{n}$ than pediatricians. These between-type differences may reflect the populations served by different types of providers. For example, in our sample, family medicine physicians and nurse practitioners reported serving fewer adolescents than pediatricians. While it is possible that pediatric and family nurse practitioners also differ in 
their HPV vaccine recommendation practices, our data did not allow us to differentiate nurse practitioners by practice specialty. Future research in this area should examine differences among nurse practitioners. Efforts to improve HPV should continue to reach out to healthcare professionals beyond pediatricians to include all providers who provide preventive care to adolescent patients.

\section{Strengths and limitations}

Study strengths include a large, statewide sample and diversity of respondents with regard to specialty. Limitations include a cross-sectional design and a modest response rate which may lead to nonresponse bias and limits the generalizability of our findings; however the cooperation rate among confirmed eligible providers was quite high. While our study is strengthened by a focus on the target age group for HPV vaccination, previous research consistently finds that providers' recommendation intentions and practices vary by patient age (Allison et al., 2013; Daley et al., 2010; Kahn et al., 2005; Weiss et al., 2010). Future research should investigate HPV vaccine hesitancy among parents of older adolescents as well as among adolescent patients themselves. Our data are based on providers' self-report which may be subject to recall and social desirability bias; however, responses were anonymous, reducing this likelihood. Findings regarding nurse practitioners may be skewed because we were not able to distinguish between pediatric and family nurse practitioners in the present study. Finally, since we surveyed healthcare providers from a single state, the generalizability of the findings to other populations in other regions will need to be established.

\section{Conclusion}

This study offers new information on healthcare providers' reported HPV vaccine recommendation practices for boys post-licensure and provides insight into healthcare providers' experiences with parental vaccine hesitancy. Increasing HPV vaccine coverage, particularly in the target age range of young adolescents, is a national health priority (U.S. Department of Health and Human Services). However, the high proportion of providers in our study who do not routinely recommend HPV vaccine to 11-12 year olds as per national guidelines, combined with providers' perceptions of widespread parental concerns about the vaccine, point to the importance of developing tools and strategies that can enable providers to effectively address HPV vaccine hesitancy (Sadaf, Richards, Glanz, Salmon, \& Omer, 2013). Intervention research is needed to speed adoption of recommended HPV vaccination practices among providers and ultimately increase uptake among young adolescents. The results of this study highlight potential targets to improve healthcare providers' recommendations and tools for more effectively counseling vaccine-hesitant parents that can inform future intervention.

\section{Acknowledgments}

This study was funded through a Young Investigator Award from the Academic Pediatric Association, supported by the Maternal and Child Health Bureau and the American Academy of Pediatrics (U04MC07853-03, PI: McRee) and further supported by: a NRSA in Primary Medical Care from HRSA (T32HP22239, PI: Borowsky); UNC Lineberger Cancer Control Education Program (R25 CA57726); and Clinical and Translational Science Institute grant support (UL1RR033183) to the University of Minnesota from the National Center for Research Resources. 
The authors thank Iris W. Borowsky, MD, PhD, for her mentorship on this project, and Rachel Eggert, MPH, for her assistance conducting the survey.

\section{References}

Allen JD, Othus MK, Shelton RC, Li Y, Norman N, Tom L, del Carmen MG. Parental decision making about the HPV vaccine. Cancer Epidemiology, Biomarkers and Prevention. 2010; 19(9): 2187-2198.

Allison MA, Dunne EF, Markowitz LE, O'Leary ST, Crane LA, Hurley LP, Beaty BL. HPV vaccination of boys in primary care practices. Academic Pediatrics. 2013; 13(5):466-474. [PubMed: 24011749]

American Association for Public Opinion Research (AAPOR). Standard Definitions: Final Dispositions of Case Codes and Outcome Rates for Surveys. 6th. AAPOR; 2009.

Bandura, A. Social Foundations of Thought and Action. Englewood Cliffs, NJ: Prentice Hall; 1986.

Cabana MD, Rand CS, Powe NR, Wu AW, Wilson MH, Abboud PAC, Rubin HR. Why don't physicians follow clinical practice guidelines? Journal of the American Medical Association. 1999; 282(15):1458-1465. [PubMed: 10535437]

Centers for Disease Control and Prevention (CDC). FDA licensure of quadrivalent human papillomavirus vaccine (HPV4, Gardasil) for use in males and guidance from the Advisory Committee on Immunization Practices (ACIP). Morbidity and Mortality Weekly Report. 2010; 59(20):630-632. [PubMed: 20508594]

Curtis CR, Yankey D, Jeyarajah J, Dorell C, Stokley S, MacNeil J, Hariri S. National and state vaccination coverage among adolescents aged 13-17 years-United States, 2012. Morbity and Mortality Weekly Report. 2013; 62(34):685-693.

Daley MF, Crane LA, Markowitz LE, Black SR, Beaty BL, Barrow J, Kempe A. Human papillomavirus vaccination practices: a survey of US physicians 18 months after licensure. Pediatrics. 2010; 126(3):425-433. [PubMed: 20679306]

Darden PM, Thompson DM, Roberts JR, Hale JJ, Pope C, Naifeh M, Jacobson RM. Reasons for not vaccinating adolescents: National Immunization Survey of Teens, 2008-2010. Pediatrics. 2013; 131(4):645-651. [PubMed: 23509163]

Dempsey AF, Abraham LM, Dalton V, Ruffin M. Understanding the reasons why mothers do or do not have their adolescent daughter vaccinated against human papillomavirus. Annals of Epidemiology. 2009; 19(8):531-538.10.1016/j.annepidem.2009.03.011 [PubMed: 19394865]

Dempsey AF, Zimet GD, Davis RL, Koutsky L. Factors that are associated with parental acceptance of human papillomavirus vaccines: a randomized intervention study of written information about HPV. Pediatrics. 2006; 117(5):1486-1493. doi:117/5/1486[pii]10.1542/peds.2005-1381. [PubMed: 16651301]

Dorell C, Yankey D, Strasser S. Parent-reported reasons for nonreceipt of recommended adolescent vaccinations, National Immunization Survey-Teen, 2009. Clinical Pediatrics. 2011; 50(12): 1116-1124. [PubMed: 21856964]

Dunne EF, Markowitz LE, Chesson H, Curtis CR, Saraiya M, Gee J, Unger ER. Recommendations on the use of quadrivalent human papillomavirus vaccine in males - Advisory Committee on Immunization Practices (ACIP). Morbity and Mortality Weekly Report. 2011; 60(50):1705-1708.

Fiks AG, Grundmeier RW, Mayne S, Song L, Feemster K, Karavite D, Bell LM. Effectiveness of decision support for families ,clinicians, or both on HPV vaccine receipt. Pediatrics. 2013; 131(6): 1114-1124. [PubMed: 23650297]

Gilkey MB, McRee AL, Brewer NT. Forgone vaccination during childhood and adolescence: Findings of a statewide survey of parents. Preventive Medicine. 2013; 56(3):202-306. [PubMed: 23295175]

Gilkey MB, Moss JL, McRee AL, Brewer NT. Do correlates of HPV vaccine initiation differ between adolescent boys and girls? Vaccine. 2012; 30(42):5928-5934. [PubMed: 22841973]

Harris PA, Taylor R, Thielke R, Payne J, Gonzalez N, Conde JC. Research electronic data capture (REDCap) - A metadata-driven methodology and workflow process for providing translational research informatics supporr. Journal of Biomedical Informatics. 2009; 42(2):377-381. [PubMed: 18929686] 
Healy CM, Montesinos DP, Middleman AB. Parent and provider perspectives on immunization: Are providers overestimating parental concerns? Vaccine. 2014; 32(5):579-584. [PubMed: 24315883]

Hughes CC, Jones AL, Feemster KA, Fiks AG. HPV vaccine decision making in pediatric primary care: a semi-structured interview study. BMC Pediatrics. 2011; 11(1):74. [PubMed: 21878128]

Kahn JA, Zimet GD, Bernstein DI, M RJ, Lan D, Huang B, Rosenthal SL. Pediatricians' intention to administer human papillomavirus vaccine: The role of practice characteristics, knowledge, and attitudes. Journal of Adolescent Health. 2005; 37:502-510. [PubMed: 16310128]

Kempe A, Daley MF, McCauley MM, Crane LA, Suh CA, Kennedy AM, Dickinson LM. Prevalence of parental concerns about childhood vaccines: the experience of primary care physicians. American Journal fo Preventive Medicine. 2011; 40(5):548-555.

Kreuter MW, Wray RJ. Tailored and targeted health communication: strategies for enhancing information relevance. American Journal of Health Behavior. 2003; 27(S3):S227-S232. [PubMed: 14672383]

Lechuga J, Swain G, Weinhardt LS. Perceived need of a parental decision aid for the HPV vaccine: Content and format preferences. Health Promotion Practice. 2012; 13(2):214-221. [PubMed: 21444922]

Markowitz LE, Dunne EF, Saraiya M, Lawson HW, Chesson H, Unger ER. Quadrivalent human papillomavirus vaccine: Recommendations of the Advisory Committee on Immunization Practices (ACIP). Morbity and Mortality Weekly Report. 2007; 56:1-24.

Opel DJ, Robinson JD, Heritage J, Korfiatis C, Taylor JA, Mangione-Smith R. Characterizing providers' immunization communication practices during health supervision visits with vaccinehesitant parents: a pilot study. Vaccine. 2012; 30(7):1269-1275. [PubMed: 22230593]

Opel DJ, Taylor JA, Mangione-Smith R, Solomon C, Zhao C, Catz S, Martin D. Validity and reliability of a survey to identify vaccine-hesitant parents. Vaccine. 2011; 29(38):6598-6605. [PubMed: 21763384]

Perkins RB, Clark JA. Providers' perceptions of parental concerns about HPV vaccination. Journal of Health Care fro the Poor and Underserved. 2013; 24(2):828-839.

Reiter PL, Brewer NT, Gottlieb SL, McRee AL, Smith JS. Parents' health beliefs and HPV vaccination of their adolescent daughters. Social Science and Medicine. 2009; 69(3):475-480. [PubMed: 19540642]

Reiter PL, Gilkey MB, Brewer NT. HPV vaccination among adolescent males: Results from the National Immunization Survey-Teen. Vaccine. 2013; 31(26):2816-2821. [PubMed: 23602667]

Reiter PL, Katz ML, Paskett ED. Correlates of HPV vaccination among adolescent females from Appalachia and reasons why their parents do not intend to vaccinate. Vaccine. 2013; 31(31):31213125. [PubMed: 23664990]

Rosenthal SL, Weiss TW, Zimet GD, Ma L, Good MB, Vichnin MD. Predictors of HPV vaccine uptake among women aged 19-26: Importance of a physician's recommendation. Vaccine. 2011; 29(5):890-895. [PubMed: 20056186]

Sadaf A, Richards JL, Glanz J, Salmon DA, Omer SB. A systematic review of interventions for reducing parental vaccine refusal and vaccine hesitancy. Vaccine. 2013; 31(40):4293-4304. [PubMed: 23859839]

U.S. Department of Health and Human Services. Healthy People 2020 Objectives: Immunization and infectious diseases. Retrieved April 8, 2013, from http://www.healthypeople.gov/2020/ topicsobjectives2020/overview.aspx?topicid=23

Vadaparampil ST, Kahn JA, Salmon D, Lee JH, Quinn GP, Roetzheim R, Giuliano AR. Missed clinical opportunities: Provider recommendations for HPV vaccination for 11-12 year old girls are limited. Vaccine. 2011; 29(47):8634-8641. [PubMed: 21924315]

Weiss TW, Zimet GD, Rosenthal SL, Brenneman SK, Klein JD. Human papillomavirus vaccination of males: attitudes and perceptions of physicians who vaccinate females. Journal of Adolescent Health. 2010; 47(1):3-11. [PubMed: 20547286]

Willis, GB. Cognitive Interviewing: A Tool for Improving Questionnaire Design. Thousand Oaks, CA: Sage Publications; 2005. 
Ylitalo KR, Lee H, Mehta NK. Health care provider recommendation, human papillomavirus vaccination, and race/ethnicity in the US national immunization survey. American Journal of Public Health. 2013; 103(1):164-169. [PubMed: 22698055] 


\section{Highlights}

- Despite guidelines, many healthcare providers are not routinely recommending HPV vaccine for 11-12 year old girls or boys.

- Healthcare providers perceive parental HPV vaccine hesitancy to be common.

- Perceptions of hesitancy may discourage providers from routinely recommending HPV vaccine.

- Providers' self-efficacy to address hesitancy may be important for improving vaccine uptake.

- Findings highlight potential tools for more effectively counseling vaccinehesitant parents. 


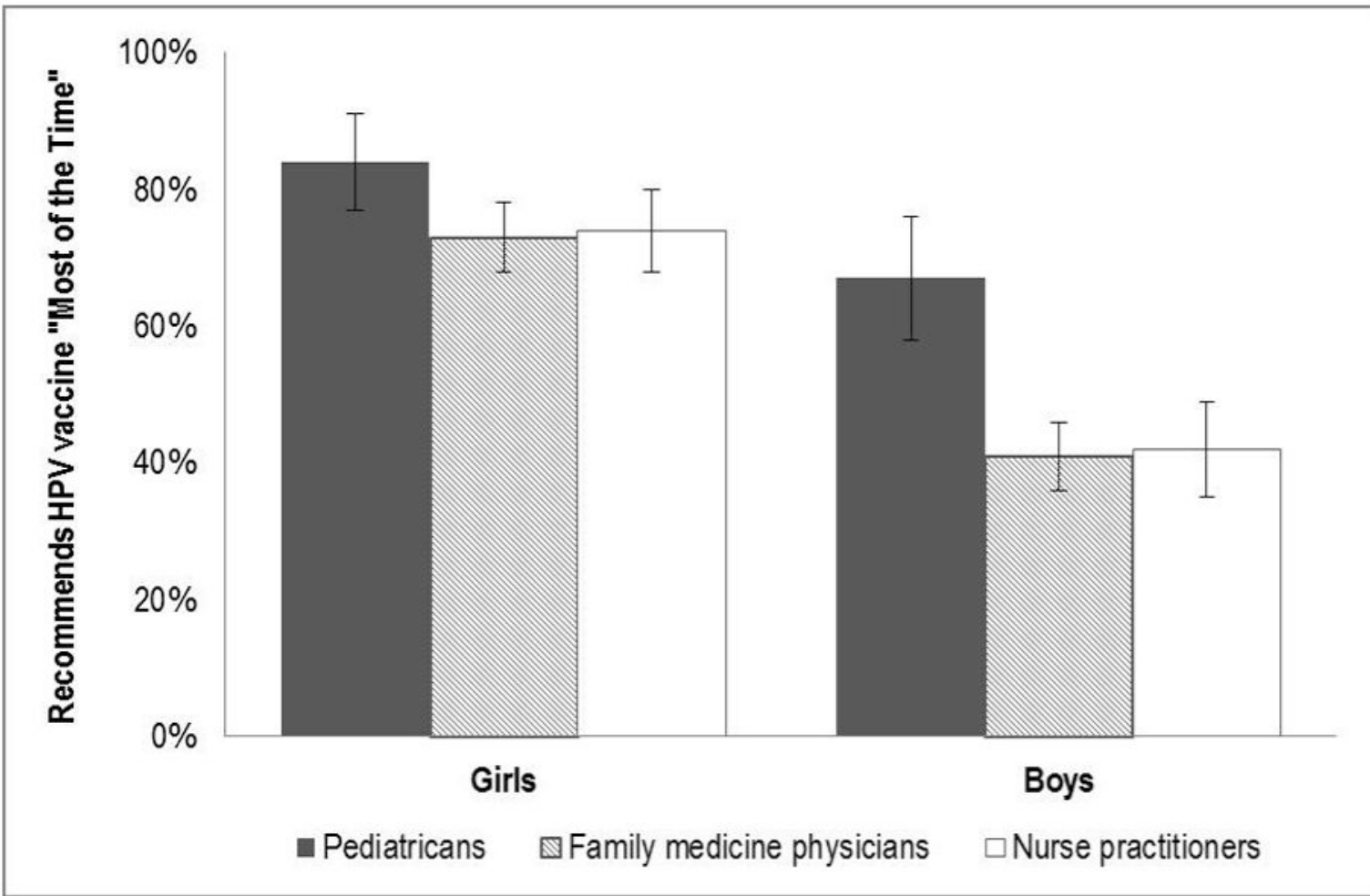

Figure 1.

Percentage of providers who recommend HPV vaccine to adolescents ages 11-12 as part of their routine care more than $75 \%$ of the time, by patient sex. Bars $=95 \%$ confidence intervals. 


\section{Table 1}

Sample characteristics $(n=575)$

\begin{tabular}{|c|c|c|}
\hline & $n$ & $(\%)$ \\
\hline \multicolumn{3}{|l|}{ Provider characteristics } \\
\hline Age in years, mean $(\mathrm{SD})^{a}$ & 48 & $(10)$ \\
\hline \multicolumn{3}{|l|}{ Sex } \\
\hline Female & 413 & $(72)$ \\
\hline Male & 162 & $(28)$ \\
\hline \multicolumn{3}{|l|}{ Training specialty } \\
\hline Pediatrician & 115 & $(20)$ \\
\hline Family medicine physician & 269 & $(47)$ \\
\hline Nurse practitioner & 191 & (33) \\
\hline \multicolumn{3}{|c|}{ Year finished residency or clinical training } \\
\hline 1989 or earlier & 147 & (26) \\
\hline 1990-1999 & 183 & $(32)$ \\
\hline $2000-2009$ & 157 & (27) \\
\hline 2010 or later & 64 & (11) \\
\hline Missing & 24 & (4) \\
\hline \multicolumn{3}{|l|}{ Practice characteristics } \\
\hline \multicolumn{3}{|l|}{ Practice type } \\
\hline Private, independent practice & 186 & $(32)$ \\
\hline Practice network / HMO & 179 & $(31)$ \\
\hline Hospital or medical center & 119 & $(21)$ \\
\hline Other & 91 & (16) \\
\hline \multicolumn{3}{|l|}{ Practice location } \\
\hline Urban & 162 & $(28)$ \\
\hline Suburban & 240 & $(42)$ \\
\hline Rural & 172 & $(30)$ \\
\hline \multicolumn{3}{|c|}{ Proportion of adolescent patients (11-17 yrs) } \\
\hline$\leq 10 \%$ & 228 & $(40)$ \\
\hline $11-25 \%$ & 209 & $(36)$ \\
\hline$>25 \%$ & 138 & $(24)$ \\
\hline
\end{tabular}

Note. Percentages may not sum to 100 due to rounding.

$a_{\text {Range }=26-82}$ years 


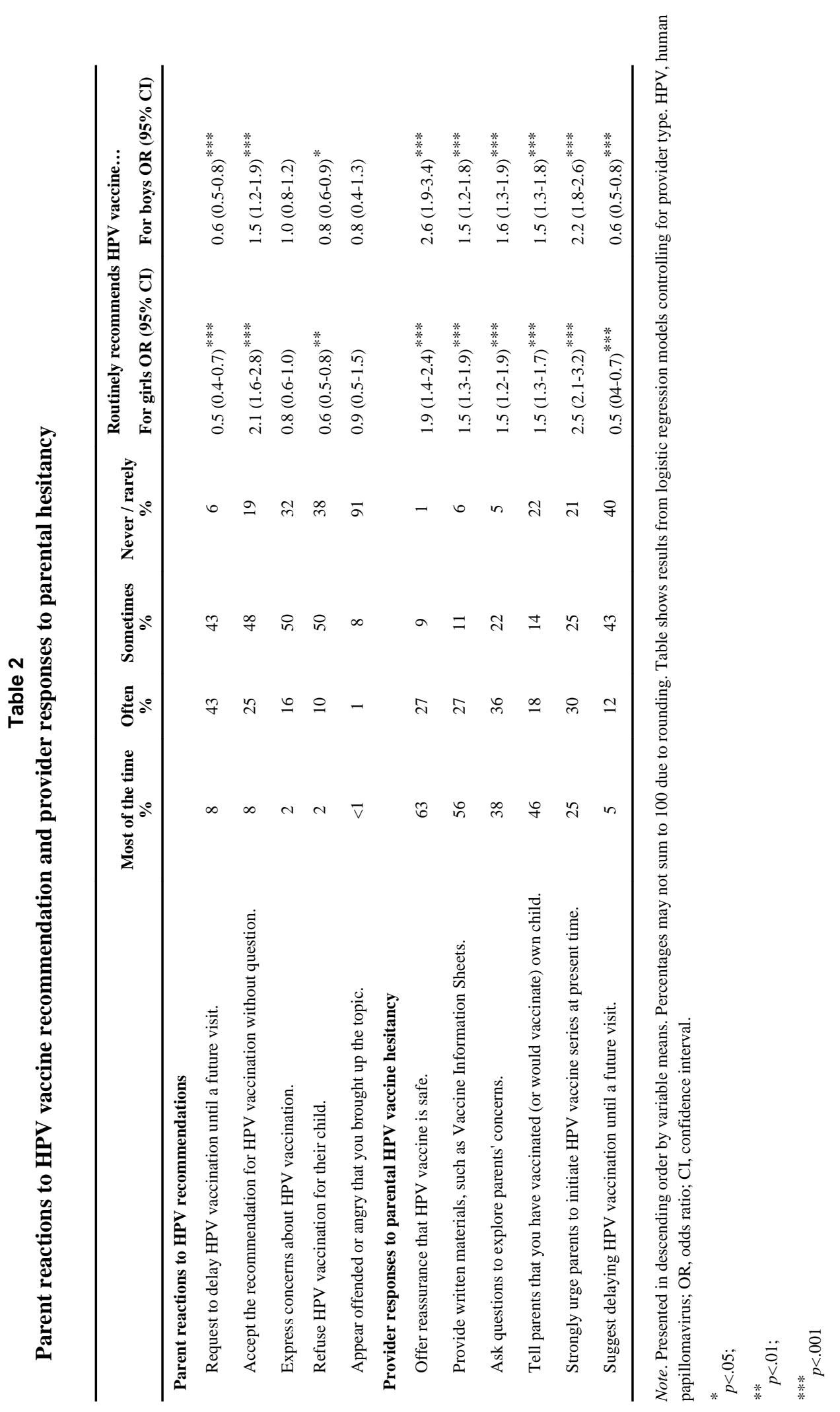

J Pediatr Health Care. Author manuscript; available in PMC 2015 November 01. 
Table 3

Providers' perception of reasons for parental HPV-vaccine hesitancy and helpfulness of potential tools

\begin{tabular}{|c|c|c|c|c|}
\hline & A lot \% & Some \% & A little \% & Not at all \% \\
\hline \multicolumn{5}{|l|}{ Perceived reasons for parental delay or refusal of HPV vaccine for their child } \\
\hline Belief that child is not sexually active & 43 & 36 & 15 & 6 \\
\hline Belief that child is unlikely to get HPV-related disease & 24 & 39 & 28 & 9 \\
\hline Discomfort talking with their child about sex & 14 & 41 & 34 & 12 \\
\hline Concerns about vaccine in general & 8 & 37 & 49 & 6 \\
\hline Concern about getting multiple vaccines & 9 & 34 & 43 & 15 \\
\hline Concern about long-term complications & 7 & 28 & 42 & 24 \\
\hline Belief that HPV is not severe & 5 & 28 & 32 & 36 \\
\hline Concern about short-term side effects (e.g., fever, pain) & 2 & 20 & 46 & 33 \\
\hline Concern about cost & 4 & 17 & 34 & 45 \\
\hline Child refused the vaccine & 2 & 15 & 37 & 46 \\
\hline Child was sick at the time of the visit & 2 & 8 & 41 & 49 \\
\hline Belief that HPV vaccine is not very effective & 1 & 10 & 30 & 59 \\
\hline \multicolumn{5}{|l|}{ Helpfulness of potential tools for counseling HPV-vaccine hesitant parents } \\
\hline Information sheets or brochures tailored to specific parent concerns & 34 & 40 & 19 & 6 \\
\hline Providing information about HPV vaccine to parents before the clinical visit & 33 & 39 & 21 & 7 \\
\hline Written information for parents that is tailored to their cultural background & 34 & 34 & 23 & 9 \\
\hline A screening questionnaire to identify specific parent concerns & 20 & 38 & 27 & 14 \\
\hline A discussion guide or script for health care professionals & 16 & 41 & 28 & 15 \\
\hline More attractive/accessible version of standard Vaccine Information Sheets & 18 & 32 & 32 & 19 \\
\hline $\begin{array}{l}\text { Interactive decision aids (e.g., written materials, computer-assisted guide) that help } \\
\text { parents make HPV vaccine decisions consistent with their values }\end{array}$ & 13 & 31 & 40 & 17 \\
\hline Providing information for parents and adolescents separately & 11 & 33 & 32 & 25 \\
\hline
\end{tabular}

Note. Presented in descending order by variable means. Percentages may not sum to 100 due to rounding. 


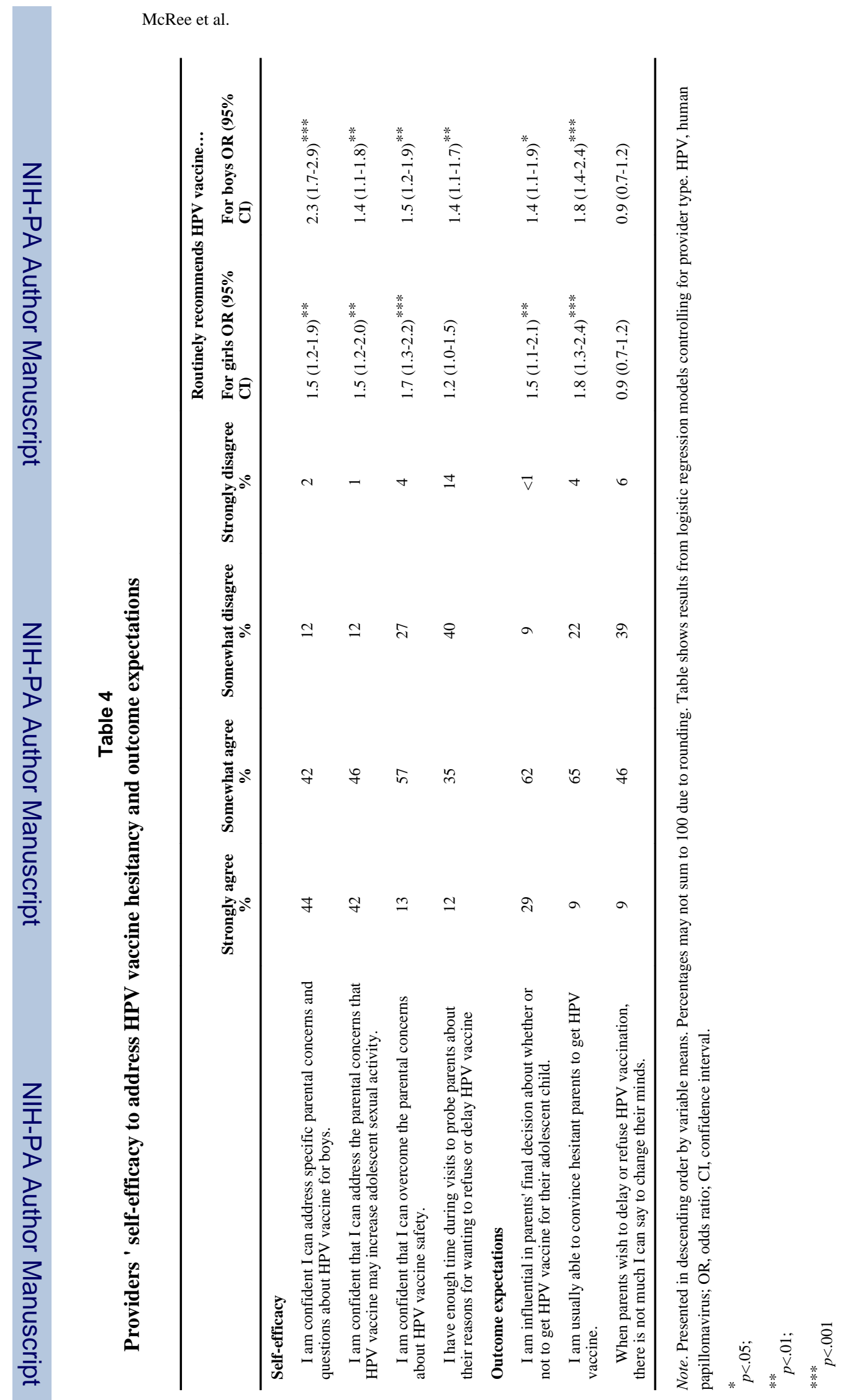

J Pediatr Health Care. Author manuscript; available in PMC 2015 November 01. 